\title{
Erratum: Póvoas et al. (2016)
}

In the article "Reliability and Construct Validity of Yo-Yo Tests in Untrained and Soccer-Trained Schoolgirls Aged 9-16" in Pediatric Exercise Science, 28(2), pp. 321-330, https://doi.org/10.1123/pes.2015-0212, the affiliation for Póvoas was incomplete, missing the university affiliation (University Institute of Maia). The online version of this article has been corrected to reflect the full affiliation for Póvoas. We apologize for this error. 